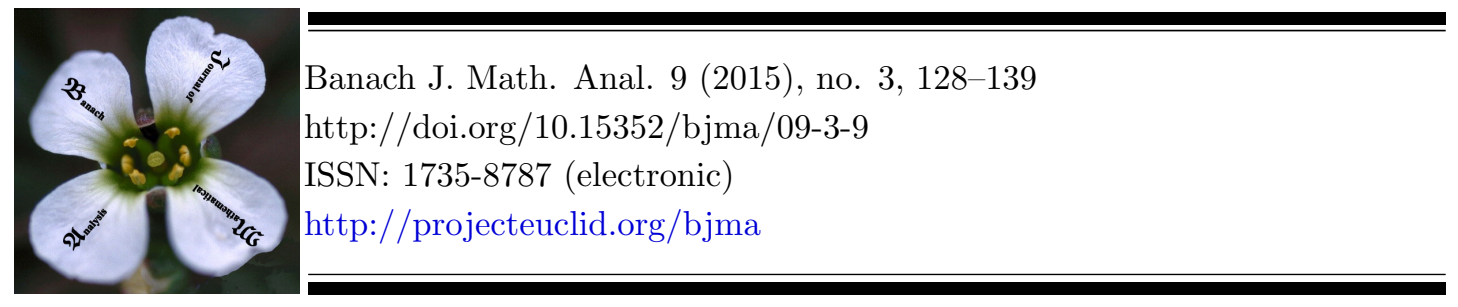

\title{
SOME COINCIDENCE AND PERIODIC POINTS RESULTS IN A METRIC SPACE ENDOWED WITH A GRAPH AND APPLICATIONS
}

\author{
DHANANJAY GOPAL ${ }^{1 *}$, CALOGERO VETRO ${ }^{2}$, MUJAHID ABBAS ${ }^{3}$ AND DEEPESH \\ KUMAR PATEL ${ }^{1}$ \\ Communicated by L. P. Castro
}

\begin{abstract}
The purpose of this paper is to obtain some coincidence and periodic points results for generalized $F$-type contractions in a metric space endowed with a graph. Some examples are given to illustrate the new theory. Then, we apply our results to establishing the existence of solution for a certain type of nonlinear integral equation.
\end{abstract}

\section{INTRODUCTION AND PRELIMINARIES}

The literature on fixed point theorems presents various cornerstone results, but certainly we can affirm that the Banach contraction mapping principle [2] plays a crucial role. In fact, this principle is one of the pivotal results of mathematical analysis and is widely considered as the source of metric fixed point theory. We say that its significance lies in its vast applicability in a number of branches of mathematics. On the other hand, this fact is a strong motivation for establishing fixed point results of mappings which satisfy certain contractive conditions and generalize the above principle; see, for instance, [4, 9, 13, 14, 16]. Recently, Ran and Reurings [12] introduced a new concept of a metric space endowed with a partial ordering and proved a fixed point theorem which generalizes Banach contraction principle. They applied this result to solve some linear and nonlinear matrix equations. In 2007, Jachymski [6] using the language of graph theory

Date: Received: Jun. 24, 2014; Accepted: Oct. 20, 2014.

* Corresponding author.

2010 Mathematics Subject Classification. Primary 47H10; Secondary 54H25, 05 C40.

Key words and phrases. Coincidence point, nonlinear integral equation, periodic point. 
(which subsumes the partial ordering) introduced the concept of $G$-contraction on a metric space endowed with a graph and proved a fixed point theorem which extends the results of Ran and Reurings [12].

In 2012, Wardowski [15] introduced the concept of $F$-contraction and proved a fixed point theorem which generalizes Banach contraction principle in many ways. Further, Abbas et al. [1] extended the results of Wardowski [15] for a pair of mappings. Most recently, Batra and Vashistha [3] combining the ideas from Jachymski [6] and Wardowski [15] introduced the concept of $F$-G-contraction and proved some interesting fixed point theorems. Following this direction of research, in this paper, we will present some coincidence and periodic points results for generalized $F$-type contractions in a metric space endowed with a graph. Consequently, several well known results are generalized and extended; also, some examples are given to support the theory. Finally, we apply our results to establishing the existence of solution for a certain type of nonlinear integral equations.

Throughout the article $\mathbb{N}, \mathbb{R}^{+}$and $\mathbb{R}$ will denote the set of natural numbers, positive real numbers and real numbers, respectively.

1.1. Graph theory. We briefly recall basic notions and notation from the literature; in particular, we refer to [6].

Let $(X, d)$ be a metric space and $\Delta=\{(x, x): x \in X\}$. Consider a graph $G$ with the set $V(G)$ of its vertices equal to $X$ and the set $E(G)$ of its edges as a superset of $\Delta$. Assume that $G$ has no parallel edges, that is $(x, y),(y, x) \in E(G)$ implies $x=y$. Also, $G$ is directed if the edges have a direction associated with them. Now we can identify the graph $G$ with the pair $(V(G), E(G))$.

Let $G^{-1}$ denote conversion of the graph $G$ obtained from the graph $G$ by reversing the direction of edges. Thus we have $V\left(G^{-1}\right)=V(G)$ and $E\left(G^{-1}\right)=$ $\{(x, y) \in X \times X:(y, x) \in E(G)\}$.

By a subgraph of $G$ we mean a graph $H$ satisfying $V(H) \subseteq V(G)$ and $E(H) \subset$ $E(G)$ such that $V(H)$ contains the vertices of all edges of $E(H)$. If $x$ and $y$ are vertices in a graph $G$ then a path in $G$ from $x$ to $y$ of length $l(l \in \mathbb{N} \cup\{0\})$ is a finite sequence $\left\{x_{n}\right\}_{n=0}^{l}$ of $l+1$ vertices such that $x_{0}=x, x_{l}=y$ and $\left(x_{i-1}, x_{i}\right) \in E(G)$ for all $i=1,2, \ldots, l$. A graph $G$ is connected if there is a path between any two vertices.

1.2. Fixed point theory. We recall some fundamental notions according to the language of graph theory.

Let $\mathcal{F}$ be the collection of all mappings $F: \mathbb{R}^{+} \rightarrow \mathbb{R}$ that satisfy the following conditions:

(F1) $F$ is strictly increasing, that is $\alpha<\beta \Rightarrow F(\alpha)<F(\beta)$ for all $\alpha, \beta \in \mathbb{R}^{+}$,

$(F 2)$ for every sequence $\left\{\alpha_{n}\right\}$ in $\mathbb{R}^{+}$we have $\lim _{n \rightarrow \infty} \alpha_{n}=0$ iff $\lim _{n \rightarrow \infty} F\left(\alpha_{n}\right)=-\infty$,

(F3) there exists a number $k \in(0,1)$ such that $\lim _{\alpha \rightarrow 0^{+}} \alpha^{k} F(\alpha)=0$.

Definition 1.1 ([15]). Let $(X, d)$ be a metric space. A mapping $f: X \rightarrow X$ is said to be an $F$-contraction on $X$ if there exists $\tau>0$ such that

$$
d(f x, f y)>0 \Rightarrow \tau+F(d(f x, f y)) \leq F(d(x, y))
$$


for all $x, y \in X$ and $F \in \mathcal{F}$.

Definition $1.2([1])$. Let $(X, d)$ be a metric space and $f, g: X \rightarrow X$. The mapping $f$ is said to be an $F$-contraction with respect to $g$ on $X$ if there exists $\tau>0$ such that

$$
\min \{d(f x, f y), d(g x, g y)\}>0 \Rightarrow \tau+F(d(f x, f y)) \leq F(d(g x, g y))
$$

for all $x, y \in X$ and $F \in \mathcal{F}$.

Remark 1.3. If $F$ is defined by $F(\alpha)=\ln \alpha$ for all $\alpha>0$, then the above contraction reduces to Jungck contraction given in [8].

Let $(X, d)$ be a metric space and denote

$$
\mathcal{G}:=\{G: G \text { is a directed graph with } V(G)=X \text { and } \Delta \subseteq E(G)\} .
$$

Definition $1.4([3])$. Let $F \in \mathcal{F}, G \in \mathcal{G}$ be given and $(X, d)$ be a metric space. A mapping $f: X \rightarrow X$ is said to be an $F$-G-contraction if

$(c 1)(x, y) \in E(G) \Rightarrow(f x, f y) \in E(G)$ for all $x, y \in X$,

$(c 2)$ there exists a number $\tau>0$ such that

$$
d(f x, f y)>0 \Rightarrow \tau+F(d(f x, f y)) \leq F(d(x, y))
$$

for all $x, y \in X$ with $(x, y) \in E(G)$.

Remark 1.5. Let $G \in \mathcal{G}$ be arbitrary and $F$ be given by $F(\alpha)=\ln \alpha$ for all $\alpha>0$. Then $F$ - $G$-contraction reduces to $G$-contraction given in [6].

Definition 1.6 ([6]). A graph $G$ is said to satisfy the property (A) if for any sequence $\left\{x_{n}\right\}$ in $V(G)$ with $x_{n} \rightarrow x$ as $n \rightarrow \infty$ and $\left(x_{n}, x_{n+1}\right) \in E(G)$ for all $n \in \mathbb{N}$ implies that $\left(x_{n}, x\right) \in G$.

Finally, let $C(f, g):=\{x \in X: f x=g x\}$ denote the set of all coincidence points of two self-mappings $f$ and $g$, defined on $X$.

\section{Coincidence POINT RESUlts}

Throughout this section we assume that $(X, d)$ is a metric space and $\mathcal{G}=\{G$ : $G$ is a directed graph with $V(G)=X$ and $\Delta \subseteq E(G)\}$.

Definition 2.1. Let $F \in \mathcal{F}$ and $G \in \mathcal{G}$. A mapping $f: X \rightarrow X$ is said to be an $F$-G-contraction with respect to $g: X \rightarrow X$ if

$\left(e_{1}\right)(g x, g y) \in E(G) \Rightarrow(f x, f y) \in E(G)$ for all $x, y \in X$, i.e. $f$ preserves edges w.r.t. $g$,

$\left(e_{2}\right)$ there exists a number $\tau>0$ such that

$$
d(f x, f y)>0 \Rightarrow \tau+F(d(f x, f y)) \leq F(d(g x, g y))
$$

for all $x, y \in X$ with $(g x, g y) \in E(G)$.

Remark 2.2. If $g=I_{X}$ (identity map on $X$ ), then the above definition reduces to Definition 1.4 given in [3]. Further, if $F$ is given by $F(\alpha)=\ln \alpha$ for all $\alpha>0$, then condition (2.1) will become Jungck $G$-contraction

$$
d(f x, f y) \leq e^{-\tau} d(g x, g y)
$$

for all $x, y \in X$ with $(g x, g y) \in E(G)$. 
Example 2.3. Let $F \in \mathcal{F}$ be arbitrary. Then every $F$-contraction w.r.t. $g$ is an $F$-G-contraction w.r.t. $g$ for the graph $G$ given by $V(G)=X$ and $E(G)=X \times X$.

Example 2.4. Let $\leq$ be a partial order on $X$. Define the graph $G$ by $E(G)=$ $\{(x, y) \in X \times X: x \leq y\}$. Then $G \in \mathcal{G}$. Also consider $F \in \mathcal{F}$ and $g=I_{X}$, a self-mapping $f: X \rightarrow X$ is an $F$-G-contraction w.r.t. $g: X \rightarrow X$ if

$\left(e_{1}\right) f$ is non-decreasing w.r.t. $\leq$,

$\left(e_{2}\right)$ there exists a number $\tau>0$ such that

$$
d(f x, f y)>0 \Rightarrow \tau+F(d(f x, f y)) \leq F(d(g x, g y))
$$

for all $x, y \in X$ with $g x \leq g y$.

We say that $f$ is an order $F$-contraction w.r.t. $g$ if $f$ and $g$ satisfy condition $\left(e_{2}\right)$ above.

Remark 2.5. Conditions $\left(e_{1}\right)$ and $\left(e_{2}\right)$ in Definition 2.1 are independent. For example, consider $f=g=I_{X}$, then the identity map always preserves edges but condition (2.1) is not satisfied for any $\alpha>0$. Further, consider $f: \mathbb{R} \rightarrow \mathbb{R}$ given by $f x=\frac{-x}{2}, x \in \mathbb{R}$ and $g=I_{\mathbb{R}}$ (identity map on $\mathbb{R}$ ), then $f$ is an order $\ln (\cdot)$-contraction w.r.t. $g$ for $\tau=\ln (2)$ but $f$ is not non-decreasing.

Now, we give an example of an $F$-G-contraction w.r.t. $g$ that is not Jungck $G$-contraction and hence not $G$-contraction.

Example 2.6. Let $S_{n}=\frac{n(n+1)}{2}, n \in \mathbb{N}$ and $X=\left\{S_{n}: n \in \mathbb{N}\right\}$. Let $d(x, y)=$ $|x-y|$ for all $x, y \in X$. Define $f, g: X \rightarrow X$ as follows:

$$
f\left(S_{n}\right)=\left\{\begin{array}{ll}
S_{n-1} & \text { if } n>1, \\
S_{1} & \text { if } n=1,
\end{array} \text { and } \quad g\left(S_{n}\right)= \begin{cases}S_{n+1} & \text { if } n>1 \\
S_{1} & \text { if } n=1 .\end{cases}\right.
$$

Also, let $G$ be a graph given by $V(G)=X$ and $E(G)=\left\{\left(S_{m}, S_{n}\right): m, n \in\right.$ $\mathbb{N}$ with $m \geq n\}$. Then, $f$ preserves edges w.r.t. $g$.

Then, we have

$$
\lim _{n \rightarrow \infty} \frac{d\left(f S_{n}, f S_{1}\right)}{d\left(g S_{n}, g S_{1}\right)}=\lim _{n \rightarrow \infty} \frac{S_{n-1}-S_{1}}{S_{n+1}-S_{1}}=1
$$

Thus $f$ is not Jungck $G$-contraction and hence not $G$-contraction. However, if we take $F(\alpha)=\ln \alpha+\alpha$ for all $\alpha>0$, then $F \in \mathcal{F}$ and $f$ is $F$-G-contraction w.r.t. $g$, putting $\tau=2$. Indeed,

$$
\begin{aligned}
\frac{d\left(f S_{n}, f S_{1}\right)}{d\left(g S_{n}, g S_{1}\right)} e^{d\left(f S_{n}, f S_{1}\right)-d\left(g S_{n}, g S_{1}\right)} & =\frac{S_{n-1}-S_{1}}{S_{n+1}-S_{1}} e^{S_{n-1}-S_{1}-S_{n+1}+S_{1}} \\
& =\frac{n^{2}-n-2}{n^{2}+3 n} e^{-2 n-1} \\
& \leq e^{-2}
\end{aligned}
$$


for all $n>1$. Also, for all $m, n \in \mathbb{N}$ with $m>n>1$, we have

$$
\begin{aligned}
\frac{d\left(f S_{m}, f S_{n}\right)}{d\left(g S_{m}, g S_{n}\right)} e^{d\left(f S_{m}, f S_{n}\right)-d\left(g S_{m}, g S_{n}\right)} & =\frac{S_{m-1}-S_{n-1}}{S_{m+1}-S_{n+1}} e^{S_{m-1}-S_{n-1}-S_{m+1}+S_{n+1}} \\
& =\frac{m^{2}+m-n^{2}-n}{m^{2}+3 m-n^{2}-3 n} e^{-2(m-n)} \\
& \leq e^{-2} .
\end{aligned}
$$

Now we state and prove our first theorem.

Theorem 2.7. Let $(X, d)$ be a metric space, $G=(V(G), E(G)) \in \mathcal{G}$ be a graph and $f, g: X \rightarrow X$ be two mappings satisfying the following conditions:

(i) there exists $x_{0} \in X$ such that $\left(g x_{0}, f x_{0}\right) \in E(G)$,

(ii) $X$ has the property (A),

(iii) $f$ is an $F$-G-contraction w.r.t. $g$,

(iv) $f(X) \subseteq g(X)$,

(v) $g(X)$ is complete.

Then $C(f, g) \neq \emptyset$.

Proof. Let $x_{0} \in X$ be as in $(i)$. Since $f(X) \subseteq g(X)$, then there exists a point $x_{1} \in$ $X$ such that $g x_{1}=f x_{0}$. From $(i)$, we have $\left(g x_{0}, f x_{0}\right) \in E(G)$, i.e., $\left(g x_{0}, g x_{1}\right) \in$ $E(G)$ and, since $f$ preserves edges w.r.t. $g$, we get $\left(f x_{0}, f x_{1}\right) \in E(G)$.

Continuing this process, having chosen $x_{n}$ in $X$, we obtain $x_{n+1}$ in $X$ such that

$$
\left(g x_{n}, g x_{n+1}\right)=\left(f x_{n-1}, f x_{n}\right) \in E(G) \text { for every } n \in \mathbb{N} \text {. }
$$

For sake of simplicity take

$$
\gamma_{n}=d\left(g x_{n}, g x_{n+1}\right) \text { for all } n \in \mathbb{N} \cup\{0\} .
$$

If there exists $n_{0} \in \mathbb{N} \cup\{0\}$ such that $g x_{n_{0}}=g x_{n_{0}+1}$, then $f x_{n_{0}}=g x_{n_{0}+1}$ implies that $f x_{n_{0}+1}=g x_{n_{0}+1}$ that is $x_{n_{0}+1} \in C(f, g)$. Now we assume $g x_{n} \neq g x_{n+1}$ for any $n \in \mathbb{N} \cup\{0\}$. Since $f$ is an $F$-G-contraction w.r.t. $g$ on $E(G)$, then we write

$$
\begin{aligned}
F\left(\gamma_{n}\right) & =F\left(d\left(g x_{n}, g x_{n+1}\right)\right) \\
& =F\left(d\left(f x_{n-1}, f x_{n}\right)\right) \\
& \leq F\left(d\left(g x_{n-1}, g x_{n}\right)\right)-\tau \\
& =F\left(d\left(f x_{n-2}, f x_{n-1}\right)\right)-\tau \\
& \leq F\left(d\left(g x_{n-2}, g x_{n-1}\right)\right)-2 \tau \\
& \leq \cdots \\
& \leq F\left(d\left(g x_{1}, g x_{2}\right)\right)-(n-1) \tau \\
& =F\left(\gamma_{1}\right)-(n-1) \tau .
\end{aligned}
$$

From

$$
F\left(\gamma_{n}\right) \leq F\left(\gamma_{1}\right)-(n-1) \tau
$$

on taking limit as $n \rightarrow \infty$, we obtain $\lim _{n \rightarrow \infty} F\left(\gamma_{n}\right)=-\infty$. Hence $\lim _{n \rightarrow \infty} \gamma_{n}=0$ by (F2). Now by (F3), there exists $k \in(0,1)$ such that $\lim _{n \rightarrow \infty} \gamma_{n}^{k} F\left(\gamma_{n}\right)=0$. 
Note that

$$
\begin{aligned}
\gamma_{n}^{k} F\left(\gamma_{n}\right)-\gamma_{n}^{k} F\left(\gamma_{1}\right) & \leq \gamma_{n}^{k}\left(F\left(\gamma_{1}\right)-(n-1) \tau\right)-\gamma_{n}^{k} F\left(\gamma_{1}\right) \\
& =-\gamma_{n}^{k}(n-1) \tau \leq 0 .
\end{aligned}
$$

Therefore, taking limit as $n \rightarrow \infty$ in (2.2), we have $\lim _{n \rightarrow \infty}(n-1) \gamma_{n}^{k}=0$.

Consequently, $\lim _{n \rightarrow \infty} n \gamma_{n}^{k}=0$. Thus there exists $n_{1}$ in $\mathbb{N}$ such that $n \gamma_{n}^{k} \leq 1$ for all $n \geq n_{1}$, that is $\gamma_{n} \leq 1 / n^{1 / k}$ for all $n \geq n_{1}$. Now for integers $m>n \geq 1$, we obtain

$$
\begin{aligned}
d\left(g x_{n}, g x_{m}\right) & \leq d\left(g x_{n}, g x_{n+1}\right)+d\left(g x_{n+1}, g x_{n+2}\right)+\cdots+d\left(g x_{m-1}, g x_{m}\right) \\
& <\sum_{i=n}^{\infty} \gamma_{i} \leq \sum_{i=n}^{\infty} \frac{1}{i^{\frac{1}{k}}}<\infty .
\end{aligned}
$$

This shows that $\left\{g x_{n}\right\}$ is a Cauchy sequence in $g(X)$. Since $g(X)$ is complete, then there exists $z \in g(X)$ such that $\lim _{n \rightarrow \infty} g x_{n}=z$. Let $w \in X$ be such that $g w=z$. By assumption $(i i)$, we have $\left(g x_{n}, z=g w\right) \in E(G)$. Hence by $(i i i)$, we get

$$
\tau+F\left(d\left(f x_{n-1}, f w\right)\right) \leq F\left(d\left(g x_{n-1}, g w\right)\right)
$$

or

$$
\begin{aligned}
F\left(d\left(g x_{n}, f w\right)\right) & =F\left(d\left(f x_{n-1}, f w\right)\right) \\
& \leq F\left(d\left(g x_{n-1}, g w\right)\right)-\tau .
\end{aligned}
$$

Since $\lim _{n \rightarrow \infty} d\left(g x_{n-1}, g w\right)=0$, then by (F2), we have $\lim _{n \rightarrow \infty} F\left(d\left(g x_{n-1}, g w\right)\right)=-\infty$. Hence $\lim _{n \rightarrow \infty} F\left(d\left(g x_{n}, f w\right)\right)=-\infty$, which implies that $\lim _{n \rightarrow \infty} d\left(g x_{n}, f w\right)=0$, i.e., $\lim _{n \rightarrow \infty} g x_{n}=f w$. Uniqueness of limit implies $z=f w=g w$, that is, $w \in C(f, g)$.

The following example illustrates Theorem 2.7.

Example 2.8. Let $X=[0,1]$ be endowed with the usual metric. Consider a graph given by $V(G)=X$ and $E(G)=\Delta \cup\left\{\left(\frac{1}{n}, \frac{1}{n+1}\right): n \in \mathbb{N}\right\} \cup\left\{\left(\frac{1}{9}, \frac{1}{3}\right)\right\} \cup$ $\left\{\left(\frac{1}{n}, 0\right): n \in \mathbb{N}\right\}$. Define $f, g: X \rightarrow X$ as follows

$$
f x=\left\{\begin{array}{ll}
\frac{1}{3} & \text { if } 0 \leq x<1, \\
\frac{1}{9} & \text { if } x=1,
\end{array} \text { and } \quad g x=x \text { for all } x \in X .\right.
$$

We note that

$$
\tau+F\left(d\left(f(1), f\left(\frac{7}{9}\right)\right)\right)=\tau+F\left(\frac{2}{9}\right) \not \leq F\left(\frac{2}{9}\right)=F\left(d\left(g(1), g\left(\frac{7}{9}\right)\right)\right)
$$

for any $\tau>0$ and any $F \in \mathcal{F}$. This means that $f$ is not an $F$-contraction w.r.t. $g$ on $X$. We will show that $f$ is an $F-G$-contraction w.r.t. $g$ on $E(G)$. Clearly, $(x, y)=(g x, g y) \in E(G)$ and $d(f x, f y)>0$ iff $x=1$ and $y \in\left\{0, \frac{1}{2}\right\}$. 
Now

and

$$
F(d(f(1), f(0)))=F\left(\left|\frac{1}{9}-\frac{1}{3}\right|\right)=F\left(\frac{2}{9}\right)<F(1)=F(d(1,0))
$$

$$
F\left(d\left(f(1), f\left(\frac{1}{2}\right)\right)\right)=F\left(\left|\frac{1}{9}-\frac{1}{3}\right|\right)=F\left(\frac{2}{9}\right)<F\left(\frac{1}{2}\right)=F\left(d\left(1, \frac{1}{2}\right)\right) .
$$

Choose any real number $\tau \in\left(0, F\left(d\left(1, \frac{1}{2}\right)\right)-F\left(d\left(f(1), f\left(\frac{1}{2}\right)\right)\right)\right)$. Then, $f$ is an $F$-G-contraction w.r.t. $g$. Also, it is easy to observe that

(i) $f(X)=\left\{\frac{1}{3}, \frac{1}{9}\right\} \subset g(X)=X=[0,1]$,

(ii) $g(X)=X=[0,1]$ is closed and hence complete,

(iii) $f$ preserves edges w.r.t. identity map $g=I_{X}$,

(iv) by the definition of $f$ and $g$, we get

$$
\left(g x_{0}, f x_{0}\right) \in E(G) \text { for } x_{0} \in\left\{\frac{1}{3}, \frac{1}{2}\right\} .
$$

Also, it is easy to see that $X$ has the property (A). Therefore, all the conditions of Theorem 2.7 are satisfied and hence $\left\{\frac{1}{3}\right\} \subseteq C(f, g)$.

\section{Periodic point Results}

It is an obvious fact that, if $f$ is a mapping which has a fixed point $x$, then $x$ is also a fixed point of $f^{n}$ for every $n \in \mathbb{N}$. However, the converse is false. Indeed, consider $X=[0,1]$ and $f: X \rightarrow X$ defined by $f x=1-x$ for all $x \in X$. Then $f$ has a unique fixed point at $x=\frac{1}{2}$, but $f^{n}=I_{X}$, for each even $n>1$, has every point of $[0,1]$ as a fixed point. On the other hand, if $X=[0, \pi]$ and $f x=\cos x$, then $f$ is nonexpansive and every iterate of $f$ has the same fixed point as $f$.

In this section we prove some periodic points results for self-mappings on a metric space $(X, d)$ endowed with a graph $G \in \mathcal{G}$. In the sequel, we need the following definition.

Definition 3.1. Let $(X, d)$ be a metric space and $f: X \rightarrow X$ be a mapping. Then $f$ is said to have the property $(\mathrm{P})$ if $F i x\left(f^{n}\right)=F i x(f)$ for every $n \in \mathbb{N}$.

For further details on these properties, we refer to [7].

Again, let $(X, d)$ be a metric space and $f: X \rightarrow X$ be a mapping. The set $\mathcal{O}(x)=\left\{x, f x, f^{2} x, \ldots, f^{n} x, \ldots\right\}$ is called the orbit of $x$.

Definition 3.2. Let $G \in \mathcal{G}$. A mapping $f: X \rightarrow X$ is called strongly orbitally $G$-continuous at $x$ if

$$
\lim _{n \rightarrow \infty} f^{n} x=p \text { and }\left(f^{n} x, f^{n+1} x\right) \in E(G) \Rightarrow \lim _{n \rightarrow \infty} f^{n+1} x=f p .
$$

A mapping $f$ is called strongly orbitally $G$-continuous on $X$ if $f$ is strongly orbitally $G$-continuous for all $x \in X$.

We denote $X^{f}:=\{x \in X:(x, f x) \in E(G)$ or $(f x, x) \in E(G)\}$.

Definition 3.3. Let $G \in \mathcal{G}$. A mapping $f: X \rightarrow X$ is called an $F$-G-graphic contraction if 
(i) $f$ preserves edges, i.e. $(x, y) \in E(G) \Rightarrow(f x, f y) \in E(G)$,

(ii) there exists a number $\tau>0$ such that

$$
d\left(f x, f^{2} x\right)>0 \Rightarrow \tau+F\left(d\left(f x, f^{2} x\right)\right) \leq F(d(x, f x))
$$

for all $x \in X^{f}$ and some $F \in \mathcal{F}$.

Remark 3.4. If we consider $F(\alpha)=\ln \alpha$ for all $\alpha>0$, then Definition 3.3 reduces to $G$-graphic contraction given in [5].

Before stating the theorem of this section, we give the following lemma without proof.

Lemma $3.5([5])$. Let $(X, d)$ be a metric space endowed with a graph $G \in \mathcal{G}$. Let $f: X \rightarrow X$ be a G-graphic contraction. Then $f$ is a $G^{-1}$-graphic contraction too.

Theorem 3.6. Let $(X, d)$ be a complete metric space and $G=(V(G), E(G)) \in \mathcal{G}$ be a graph. Suppose that $f: X \rightarrow X$ is an $F$-G-graphic contraction satisfying the following condition:

(*) $(x, f x) \in E(G)$ or $(f x, x) \in E(G)$ for all $x \in X$.

Then $f$ has the property $(P)$, provided that $f$ is strongly orbitally $G$-continuous on $X$.

Proof. Let $x_{0}$ be an arbitrary point in $X$. Define a sequence $\left\{x_{n}\right\}$ in $X$ such that $x_{n+1}=f x_{n}$ for all $n \in \mathbb{N} \cup\{0\}$ and denote $\gamma_{n}=d\left(x_{n}, x_{n+1}\right)$ for all $n \in \mathbb{N} \cup\{0\}$. If there exists $n_{0} \in \mathbb{N} \cup\{0\}$ for which $x_{n_{0}+1}=x_{n_{0}}$, then $f x_{n_{0}}=x_{n_{0}}$ and the proof is finished. Therefore, we suppose that $x_{n+1} \neq x_{n}$, for all $n \in \mathbb{N} \cup\{0\}$.

Since $\left(x_{0}, f x_{0}\right) \in E(G)$ (or $\left.\left(f x_{0}, x_{0}\right) \in E(G)\right)$ and $f$ preserves edges, then we get

$\left(f x_{0}, f\left(f x_{0}\right)\right)=\left(x_{1}, x_{2}\right)=\left(x_{1}, f x_{1}\right) \in E(G) \Rightarrow\left(f x_{1}, f\left(f x_{1}\right)\right)=\left(x_{2}, x_{3}\right) \in E(G)$.

Continuing this process, we get that $\left(f^{n} x_{0}, f^{n+1} x_{0}\right)=\left(x_{n}, x_{n+1}\right) \in E(G)$ for all $n \in \mathbb{N} \cup\{0\}$. Now using (3.1), we obtain

$$
\begin{aligned}
F\left(\gamma_{n}\right) & =F\left(d\left(x_{n}, x_{n+1}\right)\right) \\
& =F\left(d\left(f x_{n-1}, f^{2} x_{n-1}\right)\right) \\
& \leq F\left(d\left(x_{n-1}, f x_{n-1}\right)\right)-\tau \\
& =F\left(d\left(f x_{n-2}, f^{2} x_{n-2}\right)\right)-\tau \\
& \leq F\left(d\left(x_{n-2}, f x_{n-2}\right)\right)-2 \tau \\
& \leq \cdots \\
& =F\left(d\left(f x_{0}, f^{2} x_{1}\right)\right)-(n-1) \tau \\
& \leq F\left(d\left(x_{0}, x_{1}\right)\right)-n \tau \\
& =F\left(\gamma_{0}\right)-n \tau
\end{aligned}
$$

for every $n \in \mathbb{N} \cup\{0\}$. Therefore, proceeding as in the proof of Theorem 2.7, we get that $\left\{f^{n} x_{0}\right\}$ is a Cauchy sequence.

Since $\left\{f^{n} x_{0}: n \in \mathbb{N}\right\} \subseteq \mathcal{O}\left(x_{0}\right) \subseteq X$ and $X$ is complete, then there exists $x^{*}$ in $X$ such that $\lim _{n \rightarrow \infty} f^{n} x_{0}=x^{*}$. Since $f$ is strongly orbitally $G$-continuous on 
$X$, then $x^{*}=\lim _{n \rightarrow \infty} f^{n} x_{0}=f\left(\lim _{n \rightarrow \infty} f^{n-1} x_{0}\right)=f x^{*}$. Hence $f$ has a fixed point and Fix $\left(f^{n}\right)=$ Fix $(f)$ is true for $n=1$. Now assume that $n>1$ and suppose that $u \in F i x\left(f^{n}\right)$ but $u \notin F i x(f)$, then $d(u, f u)=\alpha>0$. By $(*)$, we have $(u, f u) \in E(G)$ or $(f u, u) \in E(G)$. If we assume $(u, f u) \in E(G)$, by (3.1), we obtain

$$
\begin{aligned}
F(\alpha) & =F(d(u, f u)) \\
& =F\left(d\left(f\left(f^{n-1} u\right), f^{2}\left(f^{n-1} u\right)\right)\right) \\
& \leq F\left(d\left(f^{n-1} u, f^{n} u\right)\right)-\tau \\
& \leq F\left(d\left(f^{n-2} u, f^{n-1} u\right)\right)-2 \tau \\
& \leq \cdots \\
& \leq F(d(u, f u))-n \tau .
\end{aligned}
$$

Thus $F(\alpha) \leq \lim _{n \rightarrow \infty}[F(d(u, f u))-n \tau]=-\infty$ and hence $F(\alpha)=-\infty$, which is a contradiction. Thus $u \in F(f)$.

\section{EXISTENCE OF SOLUTION FOR NONLINEAR INTEGRAL EQUATION}

Let $X=C([0, T], \mathbb{R})$ denote the space of all continuous functions on $[0, T]$ and, for $u \in X$, define the supremum norm $\|u\|=\sup _{t \in[0, T]}|u(t)|$. Clearly, $(X,\|\cdot\|)$ endowed with the metric $d$ defined by

$$
d(u, v)=\sup _{t \in[0, T]}\{|u(t)-v(t)|\}
$$

for all $u, v \in X$, is a complete metric space.

Now, following the idea in [11], we discuss the application of fixed point techniques to the solution of the nonlinear integral equation:

$$
x(t)=\quad-h_{2}(t)+\mu \int_{0}^{t} m_{1}(t, s) k_{1}(s, x(s)) d s+\rho \int_{0}^{T} m_{2}(t, s) k_{2}(s, x(s)) d s,
$$

where $t \in[0, T], \mu, \rho$ are real numbers, $h_{1}, h_{2} \in C([0, T], \mathbb{R})$ with $h_{1}(t) \geq h_{2}(t)$, and $m_{1}(t, s), m_{2}(t, s), k_{1}(s, x(s)), k_{2}(s, x(s))$ are continuous real valued functions in $[0, T] \times \mathbb{R}$. Next, consider the graph $G$ with $V(G)=X$ and

$$
E(G)=\{(u, v) \in X \times X: u(t) \leq v(t), \text { for all } t \in[0, T]\} .
$$

Clearly, $E(G)$ is a partial order. In particular, in [10], the authors showed that $(X, E(G))$ is regular, that is property $(\mathrm{A})$ holds true.

We will prove the following theorem.

Theorem 4.1. For each $x \in X$, define the operators:

$$
B x(t)=-h_{2}(t)+\mu \int_{0}^{t} m_{1}(t, s) k_{1}(s, x(s)) d s
$$


and

$$
S x(t)=x(t)-h_{1}(t)-\rho \int_{0}^{T} m_{2}(t, s) k_{2}(s, x(s)) d s,
$$

where $t \in[0, T], \mu, \rho$ are real numbers, $h_{1}, h_{2} \in C([0, T], \mathbb{R})$ with $h_{1}(t) \geq h_{2}(t)$, and $m_{1}(t, s), m_{2}(t, s), k_{1}(s, x(s)), k_{2}(s, x(s))$ are continuous real valued functions in $[0, T] \times \mathbb{R}$.

Suppose that the following hypotheses are satisfied:

(i) $\int_{0}^{T} \sup _{t \in[0, T]}\left|m_{i}(t, s)\right| d s=M_{i}<\infty, i \in\{1,2\}$,

(ii) for each $s \in[0, T]$ and for all $x, y \in X$ with $(S x, S y) \in E(G)$, there is $L_{i} \geq 0$ such that

$$
\left|k_{i}(s, x(s))-k_{i}(s, y(s))\right| \leq L_{i}|x(s)-y(s)|, \quad i \in\{1,2\}
$$

(iii) $\rho \int_{0}^{T} m_{2}(t, s) k_{2}\left(s, \mu \int_{0}^{s} m_{1}(s, \lambda) k_{1}(\lambda, x(\lambda)) d \lambda+h_{1}(s)-h_{2}(s)\right) d s=0$,

(iv) $(S x, S y) \in E(G)$ implies $(B x, B y) \in E(G)$ for all $x, y \in X$,

(v) there exists $x_{0} \in X$ such that

$$
x_{0} \leq h_{1}(t)-h_{2}(t)+\mu \int_{0}^{t} m_{1}(t, s) k_{1}\left(s, x_{0}(s)\right) d s+\rho \int_{0}^{T} m_{2}(t, s) k_{2}\left(s, x_{0}(s)\right) d s .
$$

Then the integral equation (4.2) has a solution, provided that $\frac{|\mu| L_{1} M_{1}}{1-|\rho| L_{2} M_{2}}<1$.

Proof. Note that the integral equation (4.2) has a solution if and only if the operators $B$ and $S$ have a coincidence point. Clearly, $B$ and $S$ are self-operators on $X$. Now, for all $x, y \in X$ with $(S x, S y) \in E(G)$, by assumptions $(i)$ and $(i i)$, we have

$$
\begin{aligned}
|B x(t)-B y(t)| & \leq|\mu| \int_{0}^{t}\left|m_{1}(t, s)\right|\left|k_{1}(s, x(s))-k_{1}(s, y(s))\right| d s \\
& \leq|\mu| \int_{0}^{t} \sup _{t \in[0, T]}\left|m_{1}(t, s)\right|\left|k_{1}(s, x(s))-k_{1}(s, y(s))\right| d s \\
& \leq|\mu| \int_{0}^{t} \sup _{t \in[0, T]}\left|m_{1}(t, s)\right| L_{1}|x(s)-y(s)| d s \\
& \leq|\mu| L_{1}\|x-y\| \int_{0}^{t} \sup _{t \in[0, T]}\left|m_{1}(t, s)\right| d s \\
& \leq|\mu| L_{1} M_{1}\|x-y\| .
\end{aligned}
$$

This implies that

$$
\|B x-B y\|=\sup _{t \in[0, T]}|B x(t)-B y(t)| \leq|\mu| L_{1} M_{1}\|x-y\|,
$$


By a similar reasoning we get

$$
\begin{aligned}
& \left|\rho \int_{0}^{T} m_{2}(t, s) k_{2}(s, x(s)) d s-\rho \int_{0}^{T} m_{2}(t, s) k_{2}(s, y(s)) d s\right| \\
& \leq|\rho| \int_{0}^{T}\left|m_{2}(t, s)\right|\left|k_{2}(s, x(s))-k_{2}(s, y(s))\right| d s \\
& \leq|\rho| \int_{0}^{T} \sup _{t \in[0, T]}\left|m_{2}(t, s)\right|\left|k_{2}(s, x(s))-k_{2}(s, y(s))\right| d s \\
& \leq|\rho| \int_{0}^{T} \sup _{t \in[0, T]}\left|m_{2}(t, s)\right| L_{2}|x(s)-y(s)| d s \\
& \leq|\rho| L_{2} M_{2}\|x-y\|,
\end{aligned}
$$

which implies

$$
\sup _{t \in[0, T]}\left|\rho \int_{0}^{T} m_{2}(t, s) k_{2}(s, x(s)) d s-\rho \int_{0}^{T} m_{2}(t, s) k_{2}(s, y(s)) d s\right| \leq|\rho| L_{2} M_{2}\|x-y\| .
$$

Consequently, we note that

$$
\begin{aligned}
& \|S x-S y\| \\
& \geq\|x-y\|-\sup _{t \in[0, T]}\left|\rho \int_{0}^{T} m_{2}(t, s) k_{2}(s, x(s)) d s-\rho \int_{0}^{T} m_{2}(t, s) k_{2}(s, y(s)) d s\right| \\
& \geq\left(1-|\rho| L_{2} M_{2}\right)\|x-y\|,
\end{aligned}
$$

which implies

$$
\|x-y\| \leq \frac{1}{1-|\rho| L_{2} M_{2}}\|S x-S y\| .
$$

Finally, by merging (4.3) and (4.4), we obtain

$$
\|B x-B y\| \leq \frac{|\mu| L_{1} M_{1}}{1-|\rho| L_{2} M_{2}}\|S x-S y\| .
$$

and, since $\frac{|\mu| L_{1} M_{1}}{1-|\rho| L_{2} M_{2}}<1$, we can choose $\tau>0$ such that $e^{-\tau}=\frac{|\mu| L_{1} M_{1}}{1-|\rho| L_{2} M_{2}}$ and hence, in view of (4.1), we deduce that

$$
d(B x, B y) \leq e^{-\tau} d(S x, S y)
$$

By passing to logarithms, we can write this as

$$
\ln (d(B x, B y)) \leq \ln \left(e^{-\tau} d(S x, S y)\right)
$$

and, after routine calculations, we get

$$
\tau+\ln (d(B x, B y)) \leq \ln (d(S x, S y)),
$$

for all $x, y \in X$ such that $(S x, S y) \in E(G)$. Thus, condition (2.1) is trivially satisfied. Next, adopting the same reasoning in [11], we can show that $B(X) \subseteq$ 
$S(X)$. Indeed, by $(i i i)$, for $x(t) \in X$ we have

$$
\begin{aligned}
& S\left(B x(t)+h_{1}(t)\right) \\
& =B x(t)+h_{1}(t)-h_{1}(t)-\rho \int_{0}^{T} m_{2}(t, s) k_{2}\left(s, B x(s)+h_{1}(s)\right) d s \\
& =B x(t)-\rho \int_{0}^{T} m_{2}(t, s) k_{2}\left(s, \mu \int_{0}^{s} m_{1}(s, \lambda) k_{1}(\lambda, x(\lambda)) d \lambda+h_{1}(s)-h_{2}(s)\right) d s \\
& =B x(t) .
\end{aligned}
$$

Clearly, hypothesis $(i v)$ means that $B$ preserves edges w.r.t. $S$. Next, by hypothesis $(v)$ we get

$$
x_{0}-h_{1}(t)-\rho \int_{0}^{T} m_{2}(t, s) k_{2}\left(s, x_{0}(s)\right) d s \leq h_{2}(t)+\mu \int_{0}^{t} m_{1}(t, s) k_{1}\left(s, x_{0}(s)\right) d s,
$$

that is $\left(S x_{0}, B x_{0}\right) \in E(G)$. Thus all the conditions of Theorem 2.7 are satisfied and hence its conclusion holds true, that is $B$ and $S$ have at least a coincidence point. Consequently, the integral equation (4.2) has a solution in $X$.

Acknowledgement. All authors contributed equally and significantly in writing this article. All authors read and approved the final manuscript. The first author is supported by CSIR, Govt. of India, grant number 25(0215)/13/EMR-II and Calogero Vetro is member of the Gruppo Nazionale per l'Analisi Matematica, la Probabilità e le loro Applicazioni (GNAMPA) of the Istituto Nazionale di Alta Matematica (INdAM).

\section{REFERENCES}

1. M. Abbas, B. Ali and S. Romaguera, Fixed and periodic points of generalized contractions in metric space, Fixed Point Theory Appl. 2013 (2013), article no. 243.

2. S. Banach, Sur les opérations dans les ensembles abstraits et leur application aux équations intégrales, Fund. Math. 3 (1922), no. 1, 133-181.

3. R. Batra and S. Vashistha, Fixed points of an F-contraction on metric spaces with a graph, Int. J. Comput. Math. 91 (2014), no. 12, 2483-2490.

4. A. Branciari, A fixed point theorem for mappings satisfying a general contractive condition of integral type, Int. J. Math. Math. Sci. 29 (2002), no. 9, 531-536.

5. C. Chifu and G. Petruşel, Generalized contractions in metric space endowed with a graph, Fixed Point Theory Appl. 2012 (2012), article no. 161.

6. J. Jachymski, The contraction principle for mappings on a metric space with a graph, Proc. Amer. Math. Soc. 136 (2008), no. 4, 1359-1373.

7. G.S. Jeong and B.E. Rhoades, Maps for which $F(T)=F\left(T^{n}\right)$, Fixed Point Theory Appl. 6 (2005), 87-131.

8. G. Jungck, Commuting mappings and fixed points, Amer. Math. Monthly 83 (1976), no. 4, 261-263.

9. M.S. Khan, M. Swaleh and S. Sessa, Fixed point theorems by altering distances between the points, Bull. Aust. Math. Soc. 30 (1984), no. 1, 1-9.

10. J.J. Nieto and R. Rodríguez-López, Contractive mapping theorems in partially ordered sets and applications to ordinary differential equations, Order 22 (2005), no. 3, 223-239.

11. H.K. Pathak, M.S. Khan and R. Tiwari, A common fixed point theorem and its application to nonlinear integral equations, Comput. Math. Appl. 53 (2007), no. 6, 961-971. 
12. A.C.M. Ran and M.C.B. Reurings, A fixed point theorem in partially ordered sets and some applications to matrix equations, Proc. Amer. Math. Soc. 132 (2004), no. 5, 1435-1443.

13. T. Suzuki, A generalized Banach contraction principle that characterizes metric completeness, Proc. Amer. Math. Soc. 136 (2008), no. 5, 1861-1869.

14. C. Vetro and F. Vetro, Metric or partial metric spaces endowed with a finite number of graphs: A tool to obtain fixed point results, Topology Appl. 164 (2014), 125-137.

15. D. Wardowski, Fixed points of new type of contractive mappings in complete metric spaces, Fixed Point Theory Appl. 2012 (2012), article no. 94.

16. X. Zhang, Common fixed point theorems for some new generalized contractive type mappings, J. Math. Anal. Appl. 333 (2007), no. 2, 780-786.

1 Department of Applied Mathematics \& Humanities, S.V. National Institute of Technology, Surat-395007, Gujarat, India.

E-mail address: gopaldhananjay@yahoo.in

E-mail address: deepesh456@gmail.com

2 Dipartimento di Matematica e Informatica, Università degli Studi di Palermo, Via Archirafi 34, 90123 Palermo, Italy.

E-mail address: calogero.vetro@unipa.it

${ }^{3}$ Department of Mathematics and Applied Mathematics, University of Pretoria, Lynnwood Road, Pretoria 0002, South Africa.

E-mail address: mujahid.abbas@up.ac.za 